\title{
Arthrogryposis Multiplex Congenita: A Review of Treatment Options for the Lower Extremities
}

\author{
R Byington, S Keene, E Verhovsek, J Depew
}

\begin{abstract}
Citation
R Byington, S Keene, E Verhovsek, J Depew. Arthrogryposis Multiplex Congenita: A Review of Treatment Options for the Lower Extremities. The Internet Journal of World Health and Societal Politics. 2012 Volume 7 Number 2.
\end{abstract}

\begin{abstract}
Arthrogryposis, a congenital disorder characterized by multiple joint contractures, can limit one's ability to perform even the simplest of tasks. The purpose of this paper is to outline the general limitations associated with arthrogryposis and examine the most common corrective procedures used to treat and manage deformities of the lower extremities. While the ultimate goal may be complete correction of the associated deformities, this may not be practical, as recurrence of contractures is common. Surgical and non-surgical methods discussed in this paper include casting with the Ponseti Method, use of bracing and night splinting, soft tissue release for the ankle and knee, talectomy and osteotomy procedures for the knee. The conclusions discussed in this paper determine that complete correction is not typically obtained, but quality of life can be improved through functional independence and ambulation when utilized in conjunction with thorough physical therapy rehabilitation.
\end{abstract}

\section{BACKGROUND}

Arthrogryposis, also known as Arthrogryposis Multiplex Congenita, or AMC, is a rare condition that describes congenital contractures that affect two or more different areas of the body that are present at birth. The muscles and joints affected are replaced partially, often completely, by fat and fibrous tissues (Shapiro \& Specht, 1993).

Arthrogryposis is a non-progressive disorder that is indicated by decreased or limited fetal movement of the joints and extremities before birth. To be clear, arthrogryposis is not a single disease. It has multiple etiologies, which has complicated both the understanding of the condition and results gained from treatments (Shapiro \& Specht, 1993). The exact cause of arthrogryposis is undeterminable, as studies suggest that the limitation of joint movement in-utero may be the result of a variety of neurological or muscular deficits (Cevei \& Stoicanescu, 2008). Anencephaly, spina bifida and other nerve deficiencies have proven to be neurological causes of arthrogryposis, while agenesis of muscle, fetal myopathies, myotonic dystrophy, and myasthenia gravis are just some of the muscular defects that have been linked to arthrogryposis (Cevei \& Stoicanescu, 2008). Certain skeletal defects and fetal crowding have also been shown to result in arthrogryposis. This disorder is treated by a variety of professionals, including orthopedists, geneticists, and neurologists, each of whom tends to focus on a different portion of the condition. Due to the different aspects associated with the approximately 150 different forms of AMC, the specialist involved needs to be aware of the variety of aspects of this condition in order to plan an appropriate course of treatment (Shapiro \& Specht, 1993).

"The overall prevalence of arthrogryposis is one in 3000 live births. The inheritance,

natural history, treatment guidelines, and outcomes of arthrogryposis vary among disorders, underscoring the importance of making a specific diagnosis in each child" (Bamshad, Van Heest, and Pleasure, 2009, p. 40). "Clinical studies have shown that the foot and ankle are the joints most commonly involved, but the natural history of the deformity and the best method of treatment have yet to be evaluated" (Drummond and Cruess, 1978, p. 96). According to an article by Bamshad et al. published in 2009, the most common isolated contracture is congenital clubfoot, which occurs in one of every 500 live births (Bamshad et al., 2009). With the lower extremities being the most affected by this disorder, gait dynamics and ambulation become a major functional limitation for the patient. According to Scott Giles PTA Exam: The Complete Study Guide, the normal gait cycle requires approximately $30^{\circ}$ of hip flexion, $10^{\circ}$ of hip extension, $60^{\circ}$ of knee flexion, $10^{\circ}$ of ankle dorsiflexion, and $20^{\circ}$ of ankle plantar flexion for a normal gait pattern to be observed (Giles, 2010). The contractures associated with 
AMC cause the patient's range of motion to fall outside of these norms, which leads to gait abnormalities. A couple of the common gait abnormalities that may be observed due to an extension contracture within the knee include a circumducted gait pattern, where the affected leg begins near midline and swings out to the side before returning to midline, and a vaulting gait pattern, where the uninvolved leg rises up on its toes to compensate for the extended knee position (Lippert, 2006).

\section{TREATMENT OPTIONS FOR CORRECTION AND MANAGEMENT}

The goals for treatment of arthrogryposis, no matter the severity of the condition, are geared towards increasing the functional use of the involved joints and optimizing independence in performing activities of daily living for each patient (Bernstein, 2002). Furthermore, "the aim of treatment for those cases with involvement of the lower limbs is to obtain stability, and as good weight-bearing alignment and locomotion as possible" (Mackenze, 1958, p. 18). When developing treatment plans for someone diagnosed with arthrogryposis affecting the lower extremities, interventions are usually targeted at the distal joint affected, usually the feet and ankles, and progress proximally (Bernstein, 2002). Some key parameters that should be considered when choosing the best therapeutic option include: the patient's age, contracture severity, the presence of surrounding contractures, duration of the condition, muscular development, gait pattern, the presence of an articular subluxation, radiological findings, willingness of the patient to cooperate, and availability of health care (Tepeneu et al., 2010). It is important to realize and understand that each diagnosis may differ; therefore, each treatment is patient specific and may not produce the same result for other patients. While arthrogryposis can affect multiple joints of the body, this report will focus on treatment options for contractures and deformities of the foot, ankle, and knee, as these are the more commonly affected joints associated with arthrogryposis.

\section{NON-SURGICAL TREATMENT OPTIONS CASTING PONSETI METHOD}

One of the primary methods of casting used for the treatment of arthrogryposis is the Ponseti Method, which is mainly used to treat a deformity known as clubfoot. The Ponseti Method gradually corrects the deformity by using serial casting and bracing to maintain the correction (Boehm,
Limpaphayom, Alaee, Sinclair, \& Dobbs, 2008). This is typically performed along with a percutaneous tenotomy, or tendon lengthening, of the Achilles tendon to correct ankle equines, a plantar-flexed position of the foot. Tenotomy is performed before the patient receives their last cast if they have less than $10^{\circ}$ of dorsi-flexion (Boehm et al., 2008). After casting, braces are then prescribed to be worn full time, followed by a period of part-time use to maintain the correction. If initiated within the first few years of life, extensive surgical procedures may be avoided (Boehm et al., 2008). In a past study on treatment for distal arthrogryposis, the Ponseti Method was successful for 11 of the 12 patients involved. At the conclusion of their treatments, they measured with an average ankle dorsi-flexion of $15^{\circ}$ bilaterally and no evidence of hind foot varus (Boehm et al., 2008). One remaining patient did require an extensive soft tissue release operation after a second relapse and at the time of the latest follow up presented with plantigrade feet, a neutral heel position, and $0^{\circ}$ of ankle dorsi-flexion. This patient's family reported continued noncompliance with brace wear that is used to maintain correction of club foot deformity. This specific patient was a prime example that noncompliance with brace wear is a critical factor leading to a possible clubfoot relapse following initial correction with the Ponseti Method (Boehm et al., 2008).

\section{QUENGEL HINGE CAST}

Knee flexion contractures, common joint contractures associated with arthrogryposis, can drastically diminish one's ability to ambulate even short distances without assistance or an assistive device. Casting has shown improvement in knee flexion in young children, but it runs a risk of creating a posterior dislocation of the tibia on the femur. "The Quengel hinge can be used to avoid this problem" (Bernstein, 2002, p. 421). The Quengel cast involves applying a below-the-knee cast with a cast cuff around the thigh and a Quengel hinge incorporated in the cast. Thick padding is applied under the cast over bony pressure points. A ring nut on the hinge is turned one to four times a day to gradually extend the knee, usually to 20 to 30 degrees of flexion (Tepeneu et al., 2010). The rotational component of the Quengel hinge is placed to correspond to the rotational components of the knee, which are the femoral condyles (Bernstein, 2002). This method, as well as similar non-invasive methods of treatment, has shown to be generally successful in the treatment of mild contractures, and are often used as adjuncts after radical soft-tissue release (Tepeneu et al., 2010). 


\section{NIGHT SPLINTING AND BRACING}

In addition to casting, which is used for correcting joint contractures, night splinting and bracing are methods used for maintaining these corrections. The main difference between casting and these alternatives are the removability of splints and braces. "Night splinting is the most effective method of preventing recurrent deformity" (Staheli, Hall, Jaffe, \& Paholke , 1998, p. 34). Night splinting is very useful with children, as it does not interfere with daytime activities such as playing and socializing (Staheli et al., 1998). The most important aspect of night splinting is comfort. Uncomfortable splints will prevent the child from sleeping and will cause irritability of the skin. Ideal splints are usually made from fiberglass and lined with foam, although plaster and plastics are also deemed adequate. Splinting is utilized throughout early childhood, often lasting until at least the age of five, and should continue to be used as long as there is a significant chance of recurrence of the deformity (Staheli et al., 1998).

Bracing is also used for maintaining corrections. Braces are worn during the day in cases where they enhance effective mobility. This process begins with the use of long leg braces, which cover the entire leg to maintain proper joint positioning. Later, short leg braces are utilized, as less coverage is needed to maintain the correction. Eventually, the patient graduates out of braces entirely once the correction is determined to be permanent (Staheli et al., 1998).

\section{ORTHOPEDIC SURGICAL PROCEDURES}

When corrections cannot be achieved and maintained through casting and bracing, surgical options may become inevitable to achieve ambulation for even the most common activities of daily living such as transferring to the bathroom or standing to brush one's teeth. Surgical options for the treatment of arthrogryposis are always a last resort, as these methods carry much greater risk than non-surgical options.

\section{CLUB FOOT DEFORMITY}

Clubfoot has been presented as one of the most common deformities associated with arthrogryposis. Clubfoot can also be one of the most disabling conditions if left untreated, as it can lead to severe deformities later in the maturation process. Mackenze (1958) found the following:

In general, the more rigid the deformity is at the start of treatment, the greater the tendency to relapse regardless of the treatment selected. This deformity, however, tends to progress with growth, and incorrect weight-bearing and contractures are a common occurrence. (p. 17)

According to multiple sources, treatment should be commenced as early as possible, with surgery being the last option due to the traumatic effects it can have on bone growth (Mackenze, 1958; Shapiro \& Specht, 1993).

\section{TALECTOMY METHOD}

With arthrogryposis, muscles fail to develop normally and are replaced by fibrous tissue (Drummond \& Cruess, 1978). The joint capsules may then become rigid and incapable of stretching. Adaptive changes within the bones occur in the deformed position and may require extensive surgical procedures, sometimes multiple procedures, to correct the deformity. With club foot deformity, incongruity of the joint surfaces, along with marked tightness of the soft tissues, may be so severe that soft tissue release procedures may be unsuccessful (Hsu, Jaffray, \& Leong, 1984). Talectomy, a surgical procedure that completely removes the talus bone of the ankle, may eliminate increased tightness of the joint capsule by creating false joints between the tibia and tarsal bones without tension (Hsu et al., 1984). The foot and ankle can be manipulated into a plantigrade position, where the entire plantar surface is in contact with the ground with this procedure. Good long-term functional results can be achieved if the position is properly maintained (Hsu et al., 1984). A past study conducted by Hsu, Jaffray, and Leong (1984), observed 15 feet affected with club foot that were treated using the talectomy procedure. Of the 15 feet treated, seven developed bony ankylosis of the tibiotarsal joints approximately five years after the talectomy. While the precise cause of the bony fusion of the tibiotarsal joint is unknown, it is thought to be a favorable sign of a permanent correction of the deformity (Hsu et al., 1984). The choice between primary talectomy and soft tissue release remains unsettled. According to Staheli et al. (1998), it is recommended "that this choice be made based on the severity of the clubfoot, the preference of the family and surgeon, and the practicality of a continuous postoperative night splinting program" (p. 70).

\section{PRIMARY SOFT TISSUE RELEASE OF THE ANKLE}

Primary soft tissue release for the correction of foot and ankle deformities associated with arthrogryposis can be performed in several different ways, and is specific to the exact condition or severity of the deformity. This procedure involves a series of incisions to the skin, muscle tendons, or 
ligaments for the purpose of either allowing the soft tissue to expand or release the tendon or ligament directly from the bone (Staheli et al., 1998). "Most primary deformities can be corrected during infancy using soft tissue procedures, which are preceded and followed by corrective casts and night splinting" (Staheli et al., 1998, p. 37).

According to a past study by Drummond and Cruess (1978), posterior release was found to be successful in cases of mild to moderate deformity. Twenty-three primary posterior releases were performed on 12 patients using a variety of methods to release the structures. "A plaster cast was then worn for a minimum of six weeks followed by upright braces with an ankle stop at 90 degrees until skeletal maturity was reached" (Drummond \& Cruess, 1978, p. 97).

The results of this study showed only two feet with complete correction, six with lasting and definitive correction, four with control of the deformity until additional surgery was possible, and eleven that were deemed failures in correcting the deformity (Drummond \& Cruess, 1978). Clearly, there is no quick fix for these deformities, as recurrence or failure is common with any procedure performed. "Although most published reports favor talectomy (Menelaus, 1971; Hsu et al., 1984; Guidera \& Drennan, 1985; Solund et al., 1991), a recent poll at a European Pediatric Orthopedic Society meeting revealed that most performed a posterior medial release primarily" (Staheli et al., 1998, p. 70).

\section{KNEE CONTRACTURES}

Although clubfoot deformity may be the most common deformity associated with arthrogryposis, contractures of the knees can present the most difficult problems for these patients. Knee contractures are classified as either flexion contractures or extension contractures, with flexion contractures being both the more common and the more disabling of the two. According to a study by Bernstein (2002), only $50 \%$ of patients with flexion contractures were able to become community walkers, meaning they were able to ambulate outside the home in minimally crowded public areas. However, every patient in this study suffering from extension contractures eventually became a community walker. Due to the problematic nature of this condition, and the high rate of recurrence, surgical options are practically a necessity in these cases.

\section{SOFT-TISSUE RELEASE}

As mentioned earlier, surgical options are a last resort when treating arthrogryposis. When all other options have been exhausted, soft-tissue release is the most common surgical procedure used for knee contractures in infants. Some of the methods of soft-tissue release used in order to obtain sufficient extension for ambulation are lengthening of the hamstrings, release of the gastrocnemius, release of the posterior cruciate ligament, and division of the posterior capsule (Bernstein, 2002; Shapiro, \& Specht, 1993). Which procedure (or multiple procedures in some cases) is used is determined by the severity of the flexion contracture. Full correction is avoided during this procedure because it could result in a stretch injury, as the popliteal artery and nerve is usually tight (Bernstein, 2002). Hypertension is another concern with this procedure, making careful blood pressure monitoring highly recommended afterwards. If elevated blood pressure occurs, decreased extension of the knee will usually correct the problem. Once the blood pressure has normalized, gradual extension can be reinitiated. Following the procedure, casting and physical therapy rehabilitation are necessary to maintain the correction. Although soft-tissue release and gradual extension facilitated by casting may result in improved knee extension, permanent improvement of the motion arc is rare (Bernstein, 2002).

Soft-tissue release procedures performed for the correction of extension contractures have shown results that are more favorable. In these cases, anterior lengthening of the quadriceps was used followed by immediate and prolonged use of continuous passive motion. These procedures have shown an increase of flexion from 10 to 70 degrees, with maintenance of correction two years later (Shapiro \& Specht, 1993). This procedure does come with the possibility of the patient needing the assistance of orthotic support, but the cases presented in one particular study reviewed by Shapiro and Specht (1993) were able to continue ambulation without braces.

\section{EXTENSION OSTEOTOMY OF THE KNEE}

Although soft-tissue release is the most common recommended surgical procedure for infants with knee contractures, extension osteotomy would be the preferred method of choice for skeletally mature individuals (Bernstein, 2002). If a knee flexion contracture of more than 15 to 20 degrees remains after alternative treatment options, an extension osteotomy may be indicated to obtain functional extension for ambulation. An extension osteotomy procedure involves removal of $1-1.5 \mathrm{~cm}$ of the distal femur as well as an anteriorly based wedge to allow relaxation of the posterior neurovascular structures. It is important to know that this procedure does not change the actual joint range motion, but rather realigns the axis of the 
joint to allow for more effective weight-bearing and braceable positioning (Shapiro \& Specht, 1993).

Unfortunately, this procedure carries a risk of neurovascular damage, which is a common risk factor associated with operative correction of knee flexion contractures (Shapiro \& Specht, 1993). As is the case in any surgical correction for arthrogryposis, this procedure must be followed up with casting and physical therapy to maintain the correction.

\section{DISCUSSION}

There is no cure for arthrogryposis. Even with effective treatment, those who suffer from this condition continue to deal with its effects their entire lives, as relapses are commonplace due to the nature of the disease. The goal of any treatment plan for arthrogryposis is not to eliminate the affliction, but rather to correct the deformity as much as possible and to improve the quality of life for the patient. At this time, the Ponseti Method has proven to be very successful. Providing the patient maintains proper brace wear during treatment, this method has shown significantly lower instances of relapse than other treatments (Boehm et al., 2008). Furthermore, the Ponseti Method eliminates longterm consequences such as foot pain and osteoarthritis due to its avoidance of extensive soft-tissue release surgery (Boehm et al., 2008). Talectomy has shown similar success in correction of the condition, but the long-term effects on quality of life are not quite so promising due to an increased risk of early onset arthritis in other parts of the afflicted foot (Hsu et al., 1984). Soft-tissue release operations are perhaps the most commonly performed surgeries for correction of deformities of both the ankle and the knee. When used in conjunction with proper casting and bracing methods, such as the Quengel hinge, this procedure has shown positive results, yet not the same levels of success as other surgical procedures. An extension osteotomy performed on the knee can enhance the applicability of braces by repositioning the axis of the afflicted joint. Proper casting and bracing procedures are the key to maintaining corrections whether surgical procedures are used or not. The patient's quality of life may be further enhanced through physical therapy, as this will increase functional use of the affected joint(s) and, over time, will foster independence to support a normal life for the patient.

\section{References}

r-0. Bamshad, M., Van Heest, A. E., \& Pleasure, D. (2009). Arthrogryposis: a review and update. Journal of Bone and Joint Surgery, 91-A(4), 40-46.

r-1. Bernstein, R. (2002). Arthrogryposis and amyoplasia. Journal of the American Academy of Orthropaedic Surgeons, 10(6), 417-424.

r-2. Boehm, S., Limpaphayom, N., Alaee, F., Sinclair, M., \& Dobbs, M. (2008). Early results of the Ponseti method for the treatment of clubfoot in distal arthrogryposis. Journal of Bone and Joint Surgery, 90(7), 1501-1507. doi:

10.2106/JBJS.G.00563

r-3. Cevei, M., \& Stoicanescu, D. (2008). Arthrogryposis case report. Analele Universit $\llbracket$ ii din Oradea, Fascicula Biologie, 15, 24-26.

r-4. Drummond, D., \& Cruess, R. (1978). The management of the foot and ankle in arthrogryposis multiplex congenita. The Journal of Bone and Joint Surgery, 60(1), 96-99. r-5. Giles, S. M. (2010). PTAExam: The complete studyguide. Scarborough, ME: Scorebuilders r-6. Hsu, L., Jaffray, D., \& Leong, J. (1984). Talectomy for club foot in arthrogryposis. Journal of Bone and Joint Surgery, 66-B(5), 694-696.

r-7. Lippert, L. S. (2006). Clinical kinesiology and anatomy (4th ed.). Philadelphia, PA: F.A Davis Company r-8. Mackenze, D.Y. (1959) Arthrogryposis multiplex congenita. Proceedings of the Royal Society of Medicine, $52,17-21$.

r-9. Shapiro, F., \& Specht, L. (1993). The diagnosis and orthopaedic treatment of childhood spinal muscular atrophy, peripheral neuropathy, friedreich ataxia, and arthrogryposis. Journal of Bone and Joint Surgery, 75-A(11), 1699-1714. r-10. Staheli, L. T., Hall, J. G., Jaffe, K. M., \& Paholke, D. O. (1998). Arthrogryposis: A text atlas. New York, NY: Cambridge Press University

r-11. Tepeneu, N. F., Tepeneu, P., Serban, M., Petrescu, C., Pop, L., Popescu, B., \& Badeti, R. (2010). Orthopaedic nonsurgical treatment in chronic haemophilic joint contractures of the knee. Timisoara Medical Journal, 60(2-3), 215-222. 


\section{Author Information}

Randy Byington, Ed.D, MBA, MT (ASCP)

East Tennessee State University

Shane Keene, DHSc, MBA, RRT-NPS, CPFT, RPSGT, RST

East Tennessee State University

Ester Verhovsek, Ed.D, RT (R) (M)

East Tennessee State University

Jessica Depew, BS, PTA

East Tennessee State University 